ندا شيخ رضازاده نيكو (دانشجوى دكترى) على نقى ضيائى * (دانشيار) دانثكدهى كثاورزى، كووه علوم و مهندسى آب، دانشكاه فردوسى مشهد

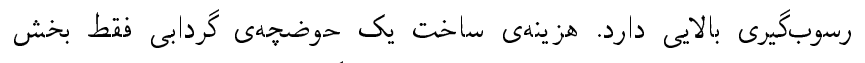

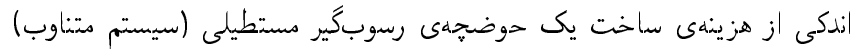

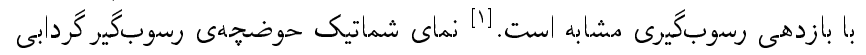

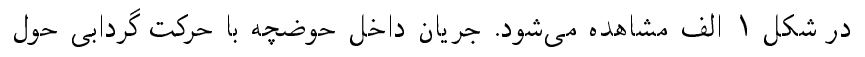

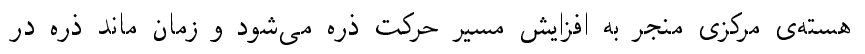

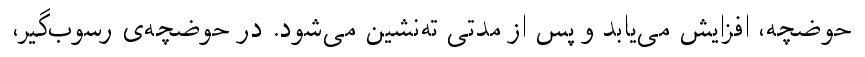

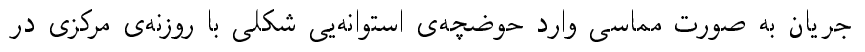

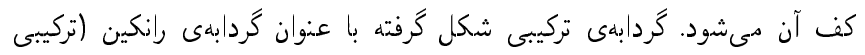

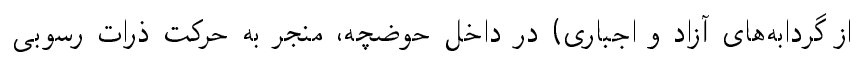

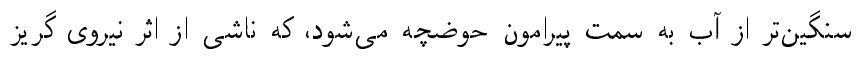

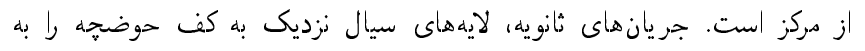

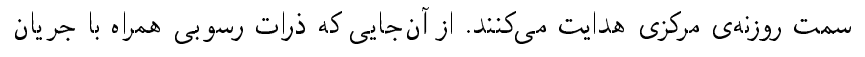

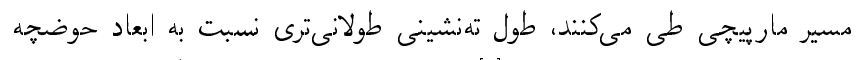

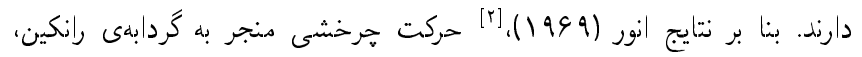

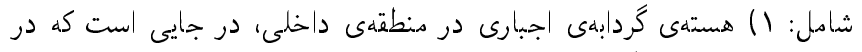

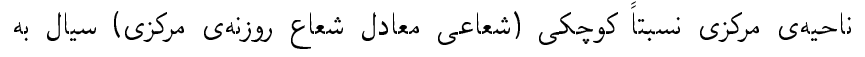

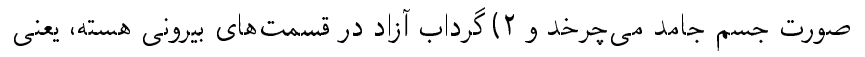

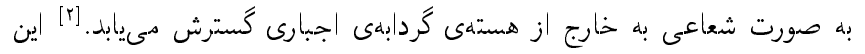

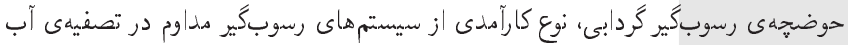

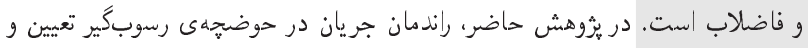

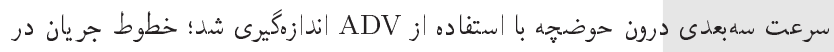
مقاطع مختلف حوضحه (در زواياى:

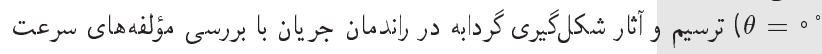

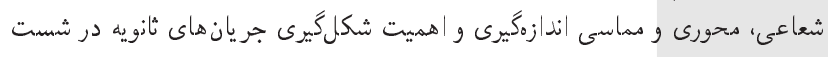

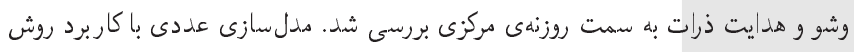

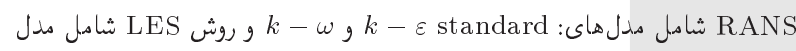

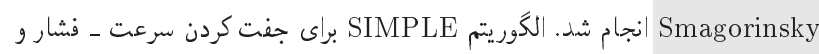

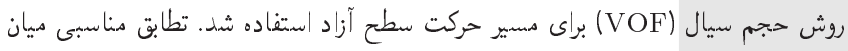

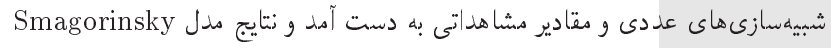

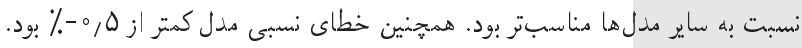

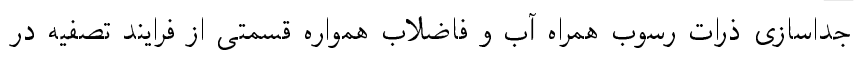

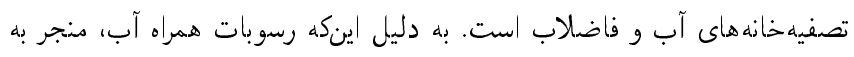

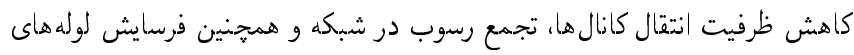

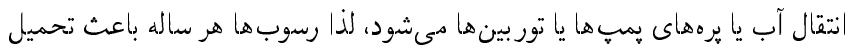

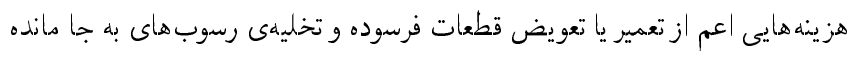

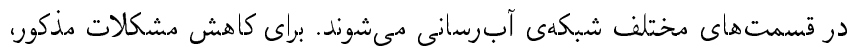

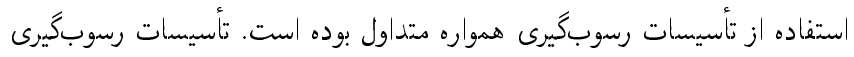

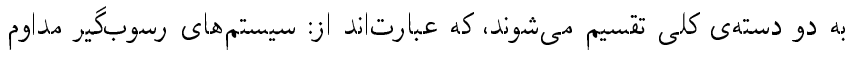

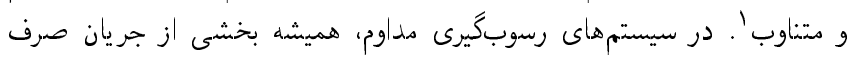

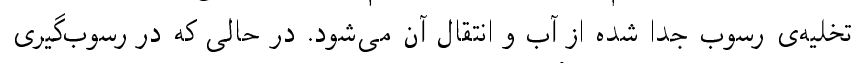

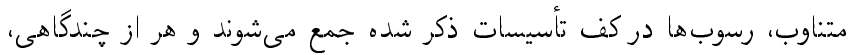

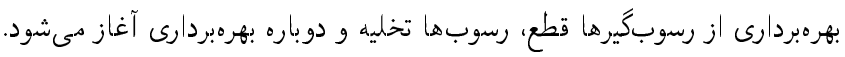

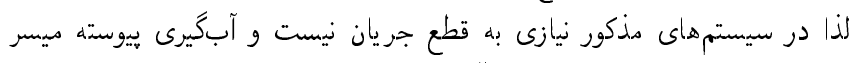

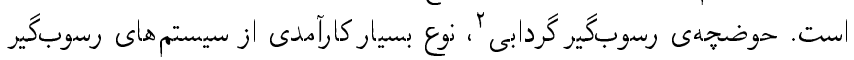

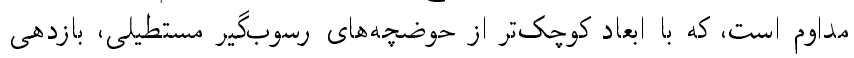

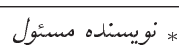

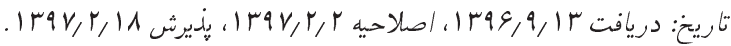
DOI:10.24200/J30.2018.5511.2247 
مى آورد. اين روش براى دو OB بيجيده در حوضجهى سادهسازى شده براى دو حالت آرام و متلاطم تكميل شده است دان.

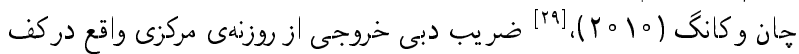

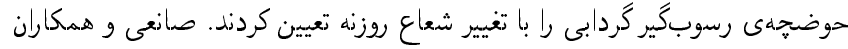

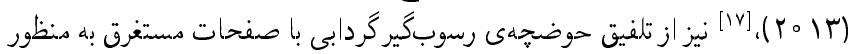

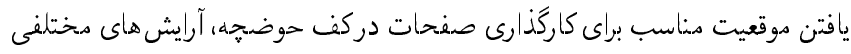

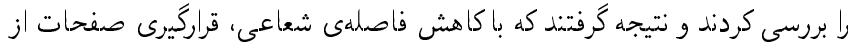

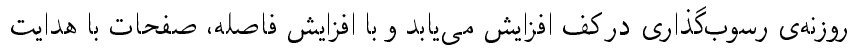

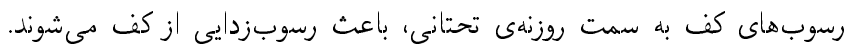

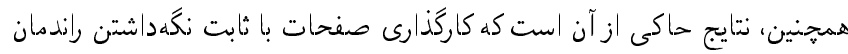

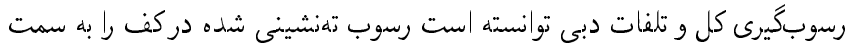

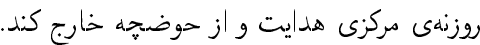

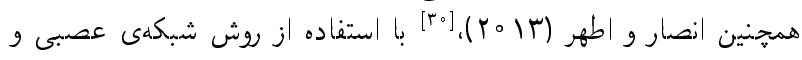

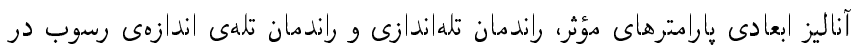

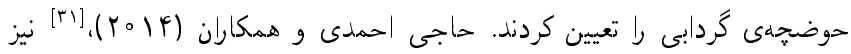

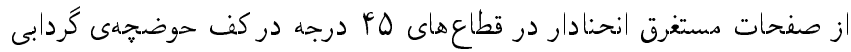

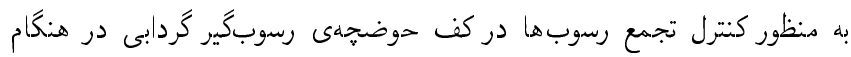

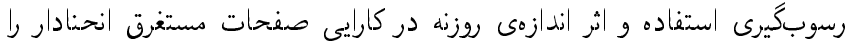

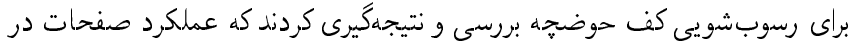

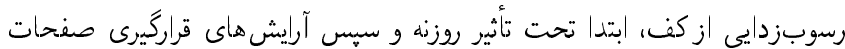

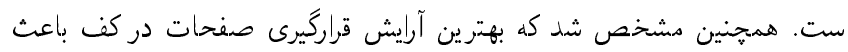

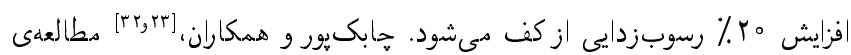

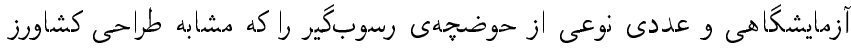

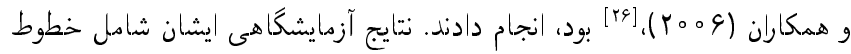

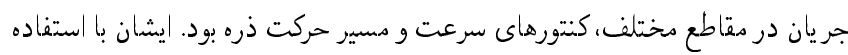

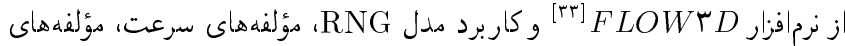

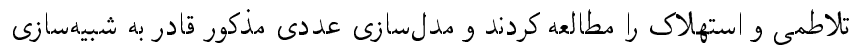

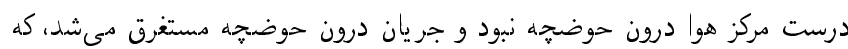

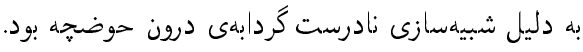

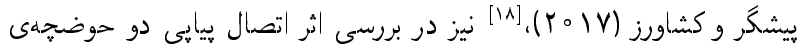

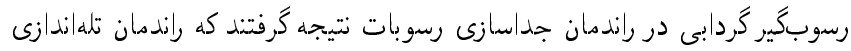

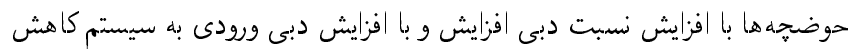

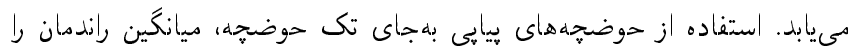

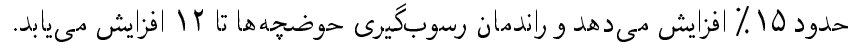

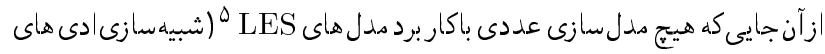

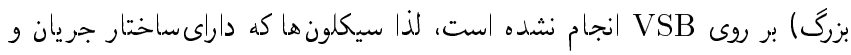

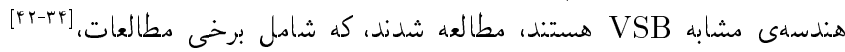

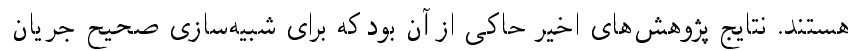

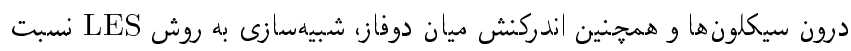

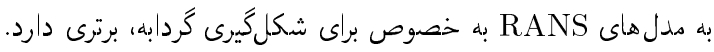

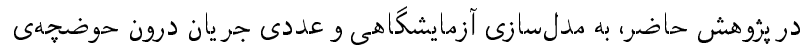

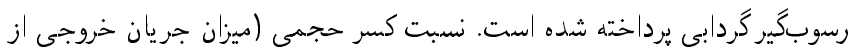

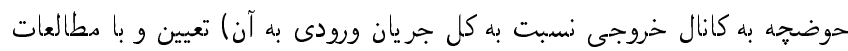

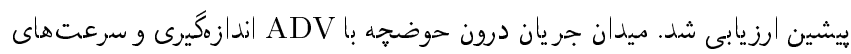

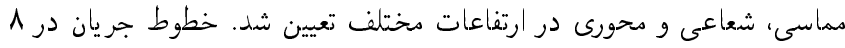

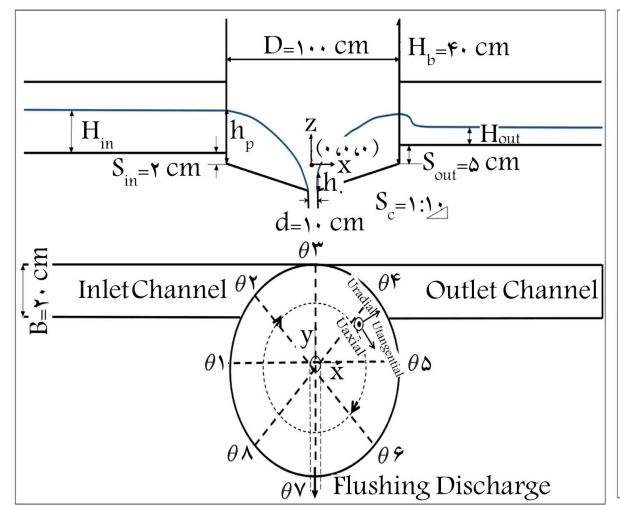

(ب)

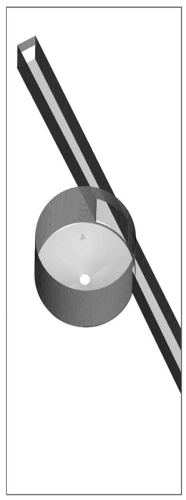

(الف)
شكل (. الف) شماى بروفيل و حوضجه و ب) يلان بعدى حوضجه رسوبكير.

ويرّى ها منجر به افزايش كارايى VSB نسبت به ساير مخازن تهنشينى معمول

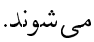

با بررسى هطالعات بيشين انجام شده بر روى حوضجههاى رسوبكير گردابى،

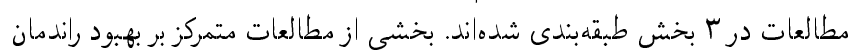

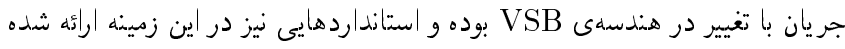

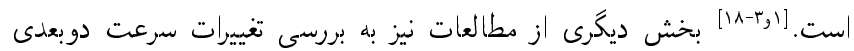

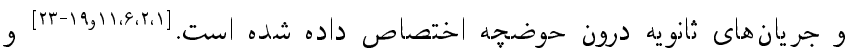

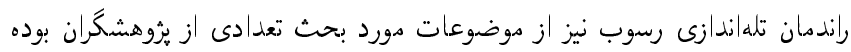

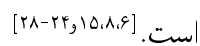

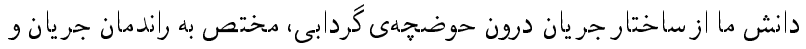

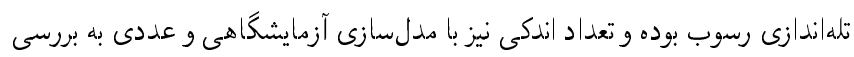

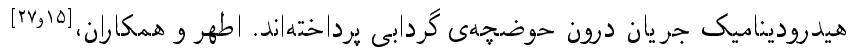

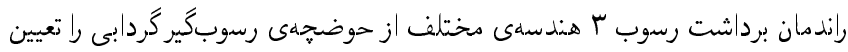

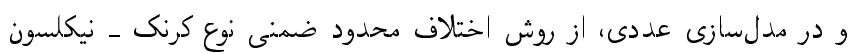

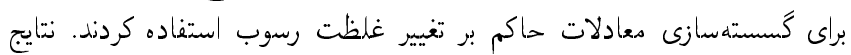

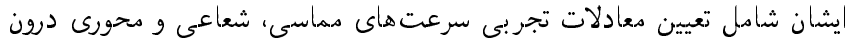

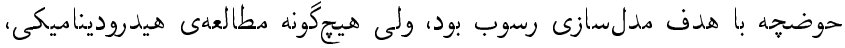

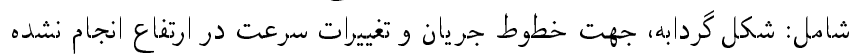

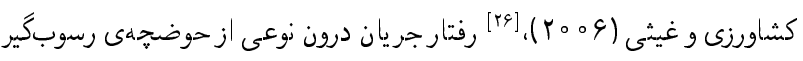

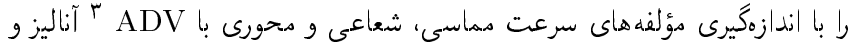

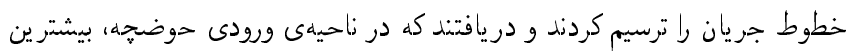

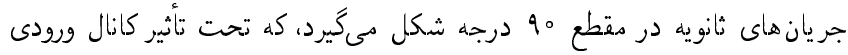

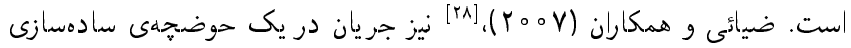

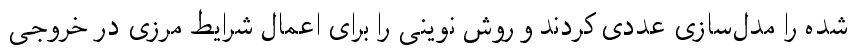

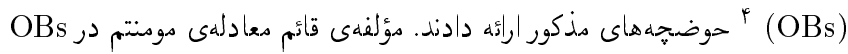

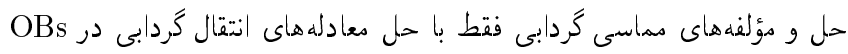

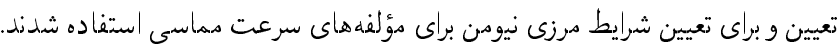

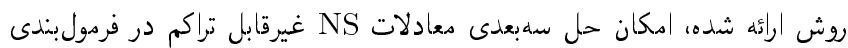

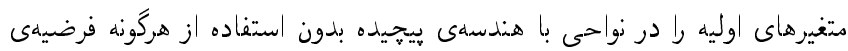

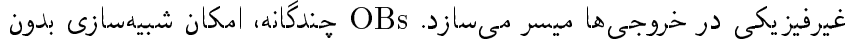

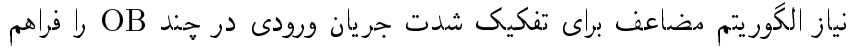




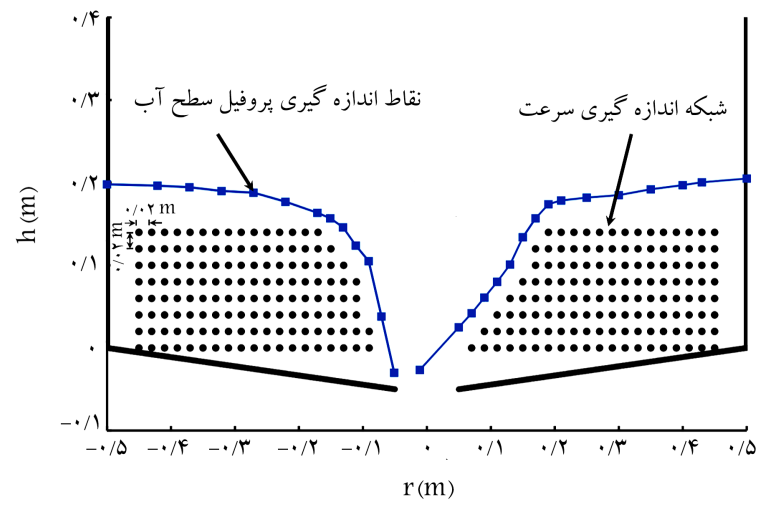

شكل ب. محل اندازگگيرى سرعت و عمق جريان.

$\frac{\partial(\rho \mathbf{u})}{\partial t}+\mathbf{u} \nabla(\rho \mathbf{u})=\nabla p+\mu \Delta \mathbf{u}+\mathbf{F}, \quad$ (الف)

$\nabla \cdot \mathbf{u}=。$

(ب)

(1)

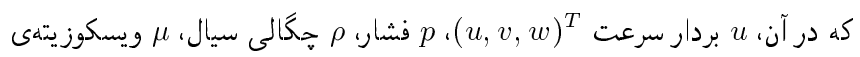

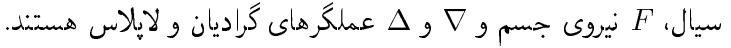

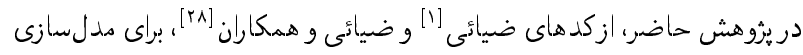

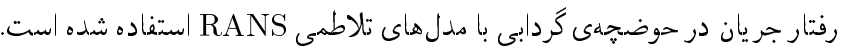

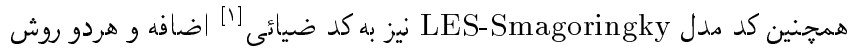
LES و RANS در Gambit تهيه و سيس دركد (زبان برنامهنويسى فورترن) فراخوانى شده است شئ.

\section{$k-\varepsilon$ standard}

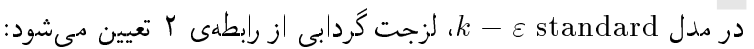

$\mu_{T}=\rho C_{\mu} \frac{k^{r}}{\varepsilon}$

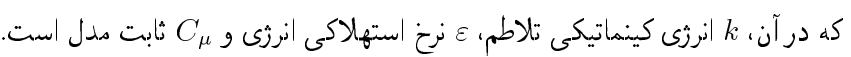

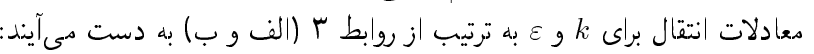
$\frac{\partial(\rho k)}{\partial t}+\nabla \cdot(\rho \mathbf{u} k)=\left(\mu+\frac{\mu_{T}}{\sigma_{k}}\right) \Delta k+G-\rho \varepsilon \quad$ (الف) $\frac{\partial(\rho \varepsilon)}{\partial t}+\nabla \cdot(\rho \mathbf{u} \varepsilon)=\left(\mu+\frac{\mu_{T}}{\sigma_{\varepsilon}}\right)$

$\Delta \varepsilon+C_{\varepsilon} \backslash \frac{\varepsilon}{k} G-C_{\varepsilon}\left\ulcorner\rho \frac{\varepsilon^{r}}{k}\right.$

كه در آن،

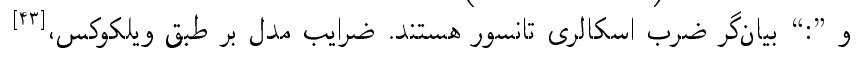
عبارتاند از: و

$C_{\mu}=\circ / \circ 9, \sigma_{k}=1 / \circ, \sigma_{\varepsilon}=1 / r, C_{\varepsilon l}=1 / 4 r, C_{\varepsilon r}=1 / 9 r$

$$
k-\omega \text { r.r. }
$$

$\mu_{T}=\rho \frac{k}{\omega}$

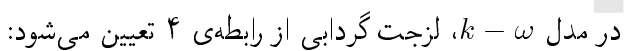

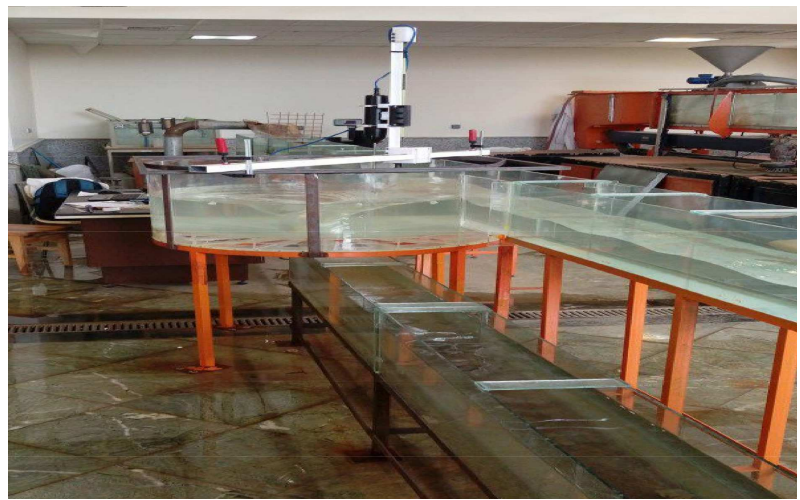

شكل r. نمايى از مدل آزمايشگاهى حوضجهى رسوبگير گردابى.

مقطع دختلف ترسيم و دسير عبور جريان در داخل حوضجه نمايش داده شده

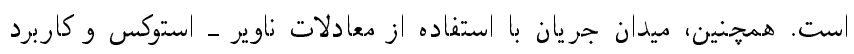
مدلهاى RANS (شامل: (Smagorinsky دقت شبيهسازىهاى بررسى شد.

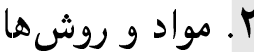

آزمايشها در آزمايشگاه دهندسى آب دانشگاه فردوسى بر روى مدل آزمايشكاهى

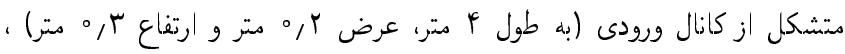

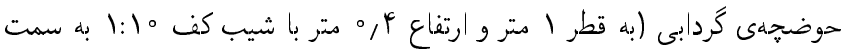

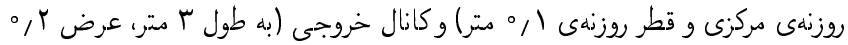

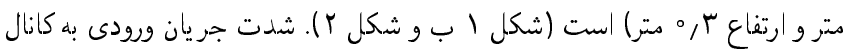

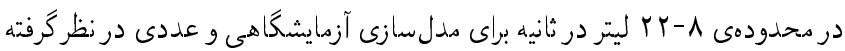

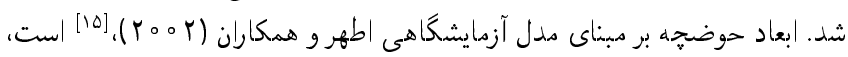

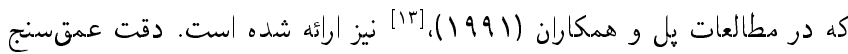

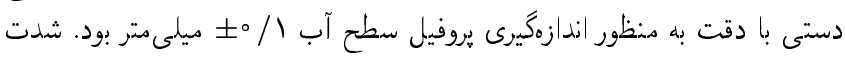

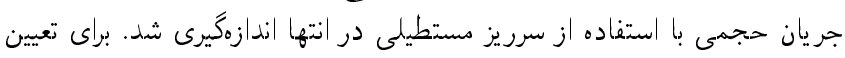

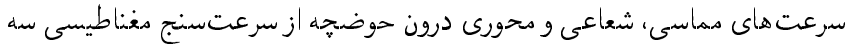
بعدى Nortek YI MHz Micro - ADV در شرايط آب زلال استفاده شد. سرعتها در م مقطع با زواياى مختلف ( )

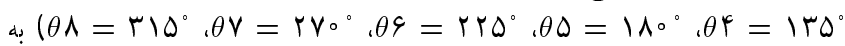

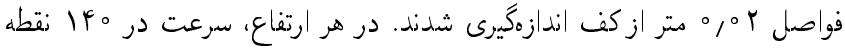

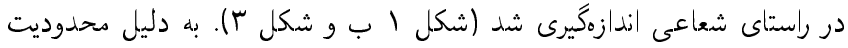

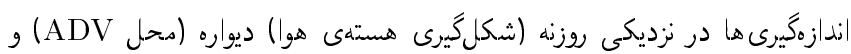

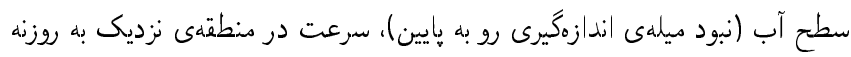

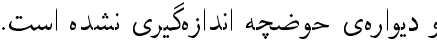
r. معادلات حركت و اجراى مدل عددى

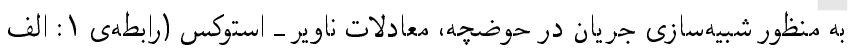

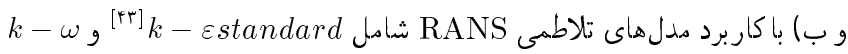

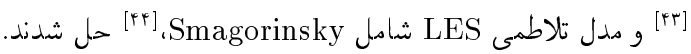




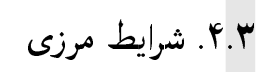

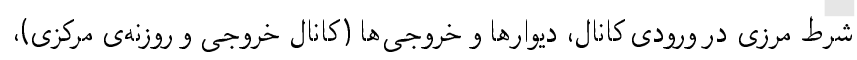

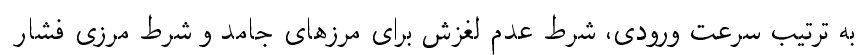

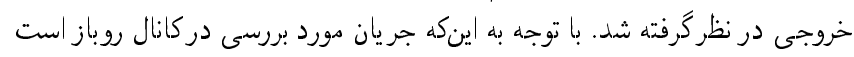

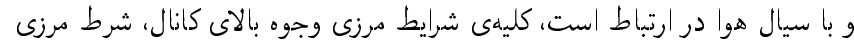

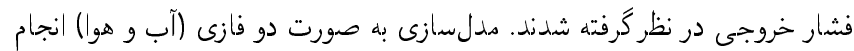
و از VOF براى شبيهسازى سطح درنه شُبن استفاده شد.

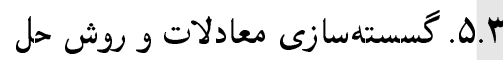

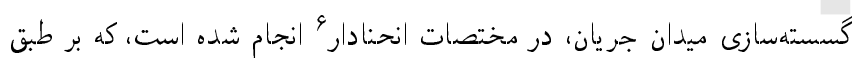

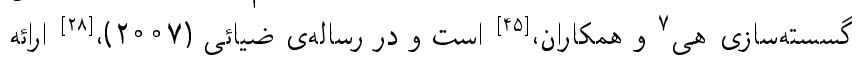

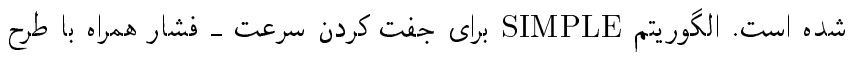

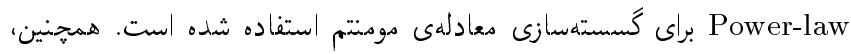
روش حجم سيال (VOF) براى مسير حركت سطح آزاد در نظر كُفته شده است.

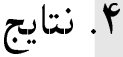

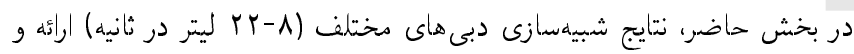

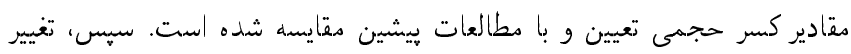

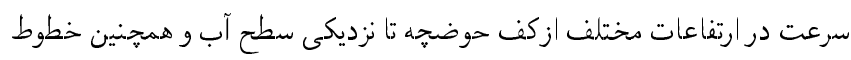

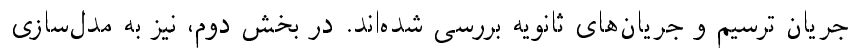

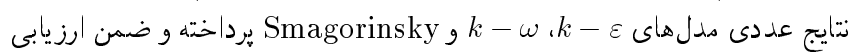

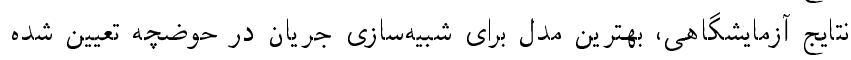

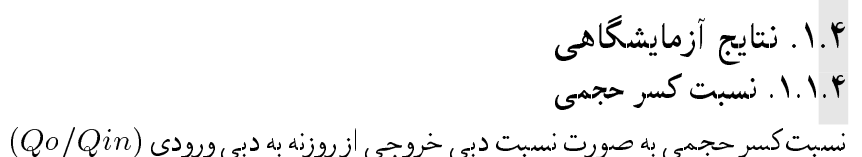

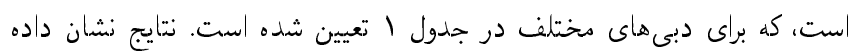

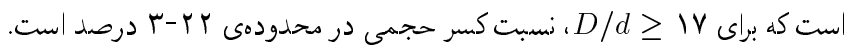

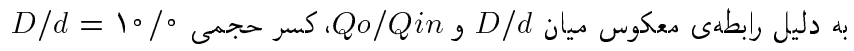

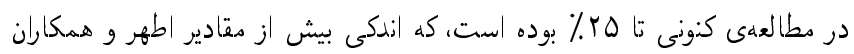

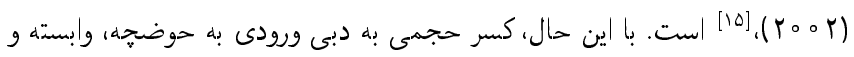

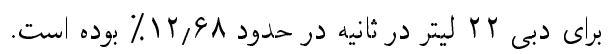

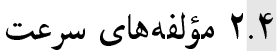

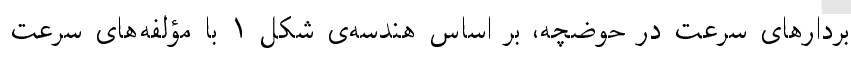

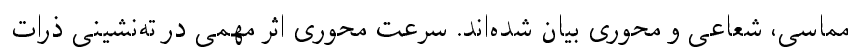

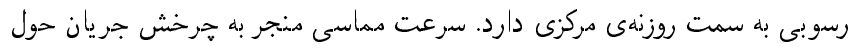

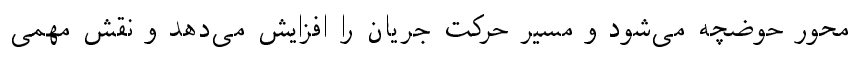

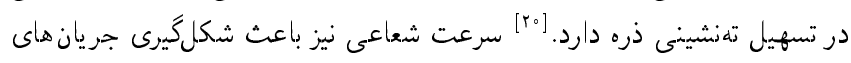

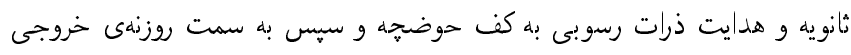

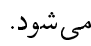

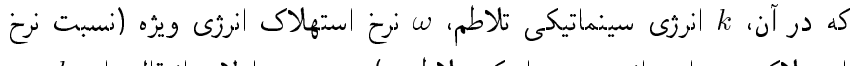

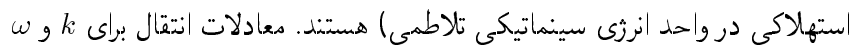
به ترتيب از روابط ه (الف و ب) به دست مى آيند:

$\frac{\partial(\rho k)}{\partial t}+\nabla(\rho \mathbf{u} k)=\left(\mu+\sigma \mu_{T}\right) \Delta k+G \beta \rho k \omega \quad$ (الف) $\frac{\partial(\rho \omega)}{\partial t}+\nabla \cdot(\rho \mathbf{u} \omega)=\left(\mu+\sigma \mu_{T}\right) \Delta \omega+\alpha \frac{\omega}{k} G-\beta \rho \omega^{r} \quad(ب)$

كه در آن، ضرايب دعادله برابرند با: $\alpha=\Delta / \uparrow, \beta=r / \uparrow^{\circ}, \beta^{*}=\circ / \circ \uparrow, \sigma=\circ / \Delta, \sigma^{*}=\circ / \Delta$

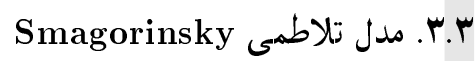
با فيلتركيرى از معادلهى مومنتم (1 ـ الف)، رابطهى 9 به دست مى آيد:

$$
(\tilde{U})_{t}+\nabla(\tilde{\mathbf{U}} \tilde{\mathbf{U}})=\nabla(\tilde{P})+\nu \Delta(\tilde{\mathbf{U}}) \nabla \cdot \tau_{S G S}
$$

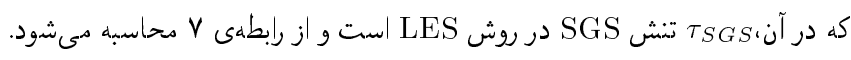

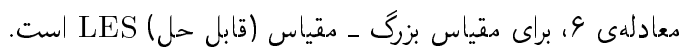

$$
\tau_{S G S, i j}=-\boldsymbol{r} \nu_{S G S} \tilde{\mathbf{S}}_{i j} \quad i, j=1, \Gamma, \Gamma \quad i \neq j
$$

$$
\text { كه در آن، }
$$

$\nu_{S G S}=l^{r}\left|\tilde{\mathbf{S}}_{i j}\right|$

$$
S_{i j}=\frac{1}{r}\left(\mathbf{u}_{i, j}+\mathbf{u}_{j, i}\right)
$$

$$
\text { مقدار بارامتر l در رابطهى } 1 \text { از رابطهى ه ا به دست مى آيد: }
$$

$l=C_{s} \Delta$

در روابط اخير،

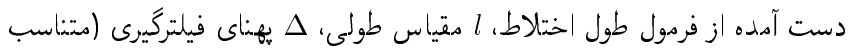

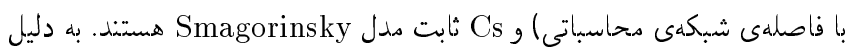

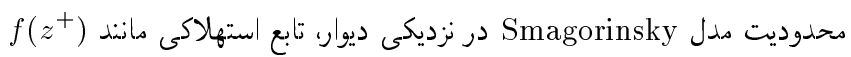

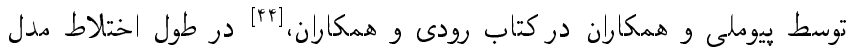
RANS

$l=C_{s} \Delta \cdot f\left(\mathbf{z}^{+}\right)$

كه در آن (f( ) از رابطهى ז ا به دست مى آيد.

$$
f\left(\mathbf{z}^{+}\right)=\sqrt{\left(1-e^{\frac{z^{+}}{A^{+}}}\right)^{r}}
$$

كه در آن، ثابت تجربى (A/0) 


\begin{tabular}{|c|c|c|c|c|}
\hline يثوهشگران & $Q_{i n}(L / s)$ & $Q_{\circ}(L / s)$ & $D / d$ & $Q_{o} / Q_{i n} \%$ \\
\hline Curi et al.( ( $9 \vee \Delta)$ & $r, \varphi \varphi-1 r, \mid r$ & $0, r r-1,1$ & $I V-V_{0}$ & $r, r-r \mid, \circ \wedge$ \\
\hline Mashauri(1919) & $\varepsilon, \Lambda-I V$ & $0,10-1,09$ & $r \Delta-r r, r r$ & $0,09-19$ \\
\hline Mashauri (1919) & $r, r r-r, 0$ & $0,10-0,11$ & $r \Delta, \Delta_{0}$ & $r, 9-8,8$ \\
\hline Mashauri (1919) & $r, r r-r, q$ & $0,10-0,11$ & $1 \Lambda-9_{0}$ & $r, r-18,9$ \\
\hline Paul et al. (19^^) & $94,0-190$ & $11, \wedge \vee-r_{0}$ & $r \wedge, r$ & $1-19$ \\
\hline Athar et al. $(r \circ r)$ & N-rt & $0,0 \wedge q-r, q$ & 10 & $v, r-I V, r$ \\
\hline Present study & $A-r r$ & $r, 1-r, \circ V$ & 10 & $\mid r, q \Lambda-r r, q \Lambda$ \\
\hline
\end{tabular}

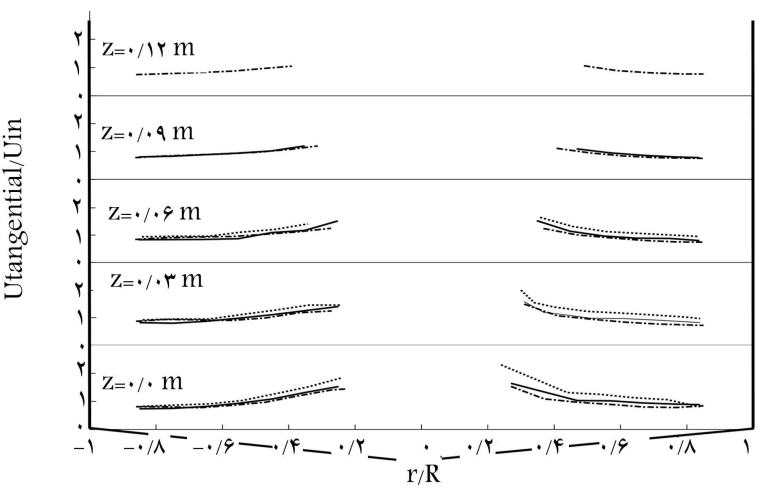

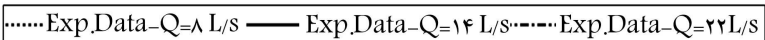

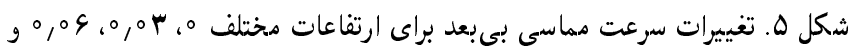

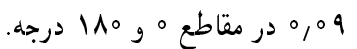

شده است. بهعلاوه، سرعت مماسى بىبعد با افزايش ارتفاع از كف حوضحه نيز كاهش يافته است. در ارتفاعات إيينتر، در نزديكى روزنه (دور از هستهى هوا)

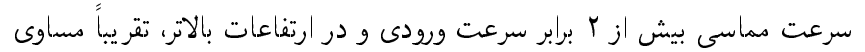

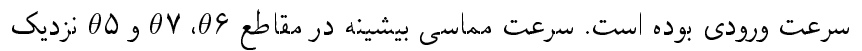
به كف است، كه دور از كانال ورودى و خروجى است. همحنين، دركليهى ارتفاعات،

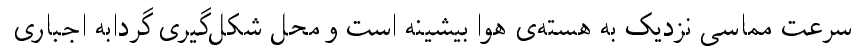

( T.Y.P

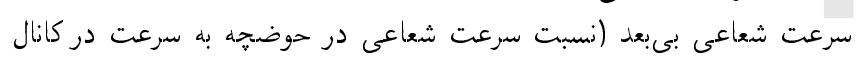

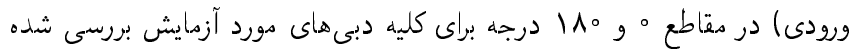

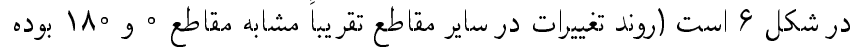

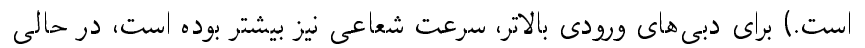

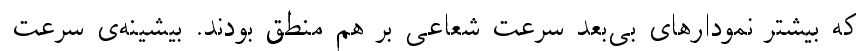

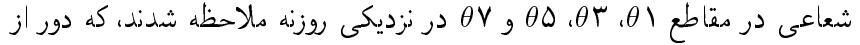

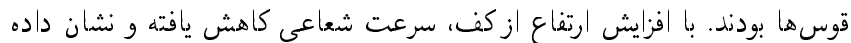

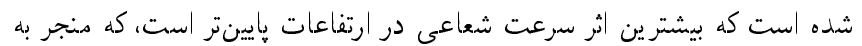
هدايت ذرات به سمت روزنه شده است.

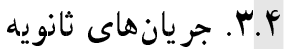

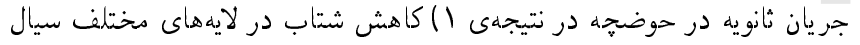

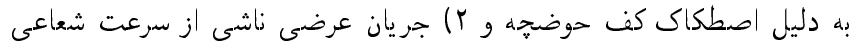

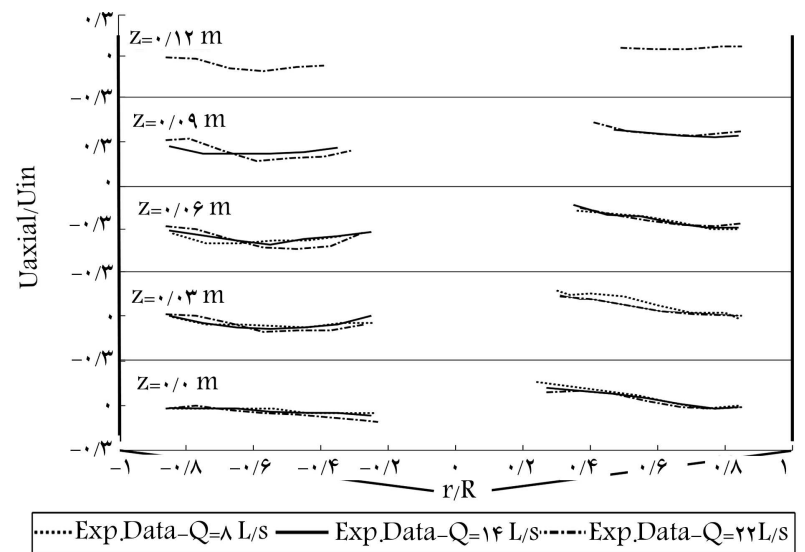

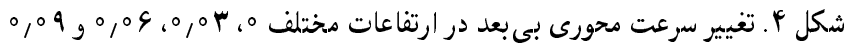

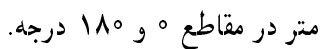

I.Y.F

سرعت دحورى بىبعد (نسبت سرعت دحورى در حوضحه به سرعت در كانال ورودى) تقريباً براى هر ب شدت جريان مشابه بوده و نسبت به سرعت مماسى

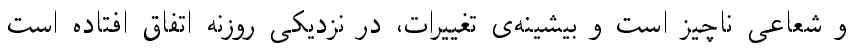

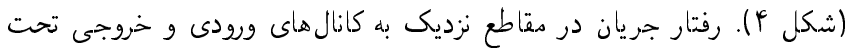
تأثير كانالهاست و در دفطع صفر درجه (سمت جهي) با توجه به ورود جريان به وريه

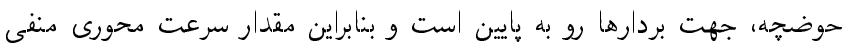

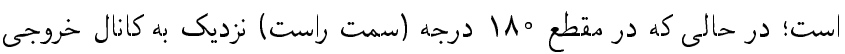
است و جريان به بيرون از كانال هدايت مىشود و بنابراين دقادير سرعت دحورى،

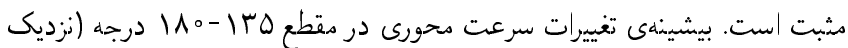

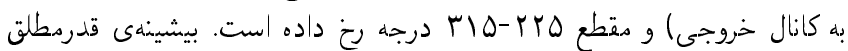
سرعت دحورى در نزديكى روزنه است، جايى كه خطوط جريان به سمت حوضهِ

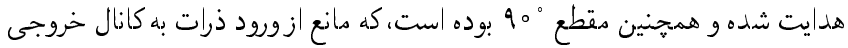
مى شود. (T.Y.P سرعت مماسى بى بعد (نسبت سرعت مماسى در حوضخه به سرعت در ورودى كانال) در شكل ه ترسيم شده است. با افزايش دبى ورودى، سرعت مماسى درون حوضحه افزايش يافته است، كه در نتيجهى افزايش نيروى سانتر يفوزى داخل حوضهِه بوده و منجر به تسهيل تهنشينى ذره شده است. در حالى كه سرعت مماسى بى بعد با افزايش دبى ورودى كاهش يافته است

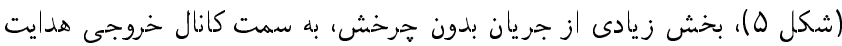




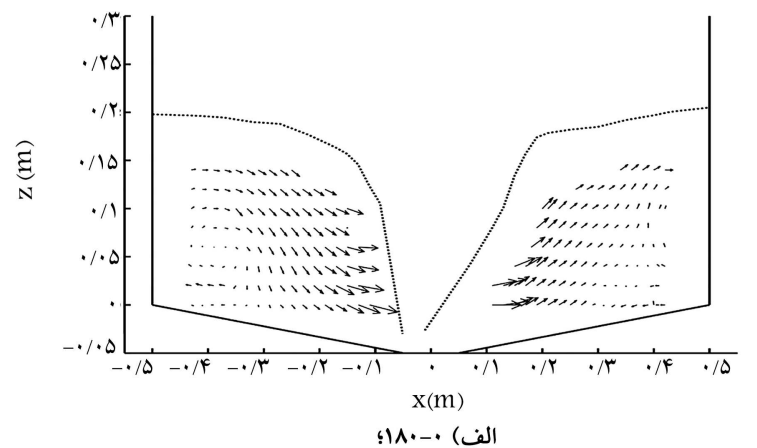

الف) (1).-.

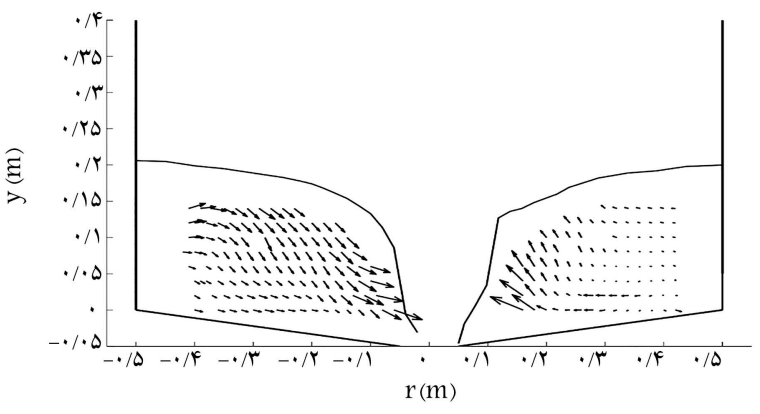

GrYA-FD (ب
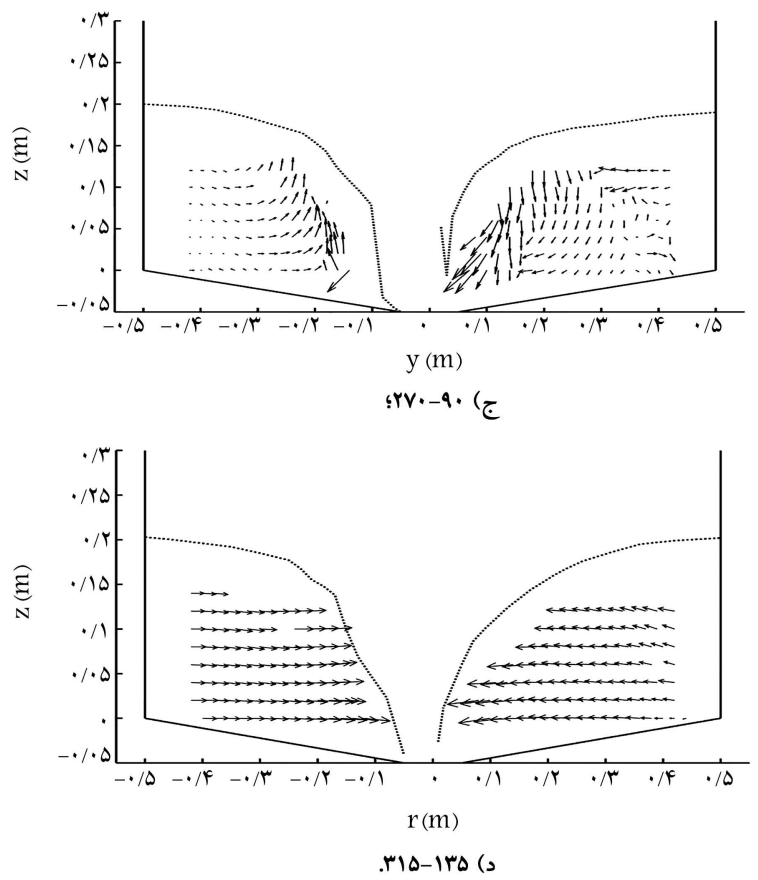

شكل v. ترسيم خطوط جر يان با دادهاى آزمايشكاهى براى مقاطع.

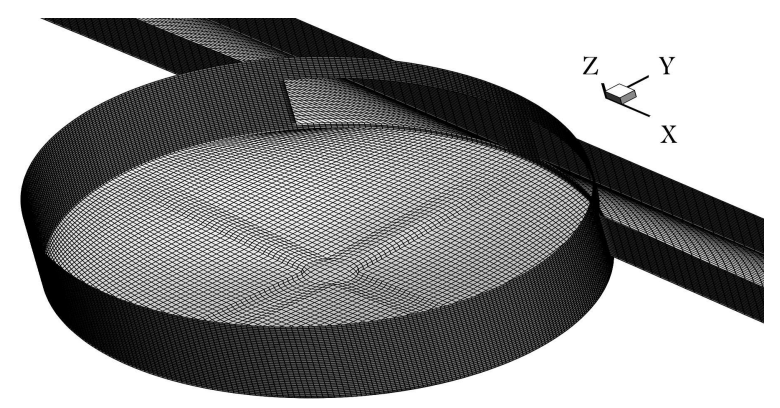

شكل ^. نمايى از شبكهى محاسباتى درون حوضحه.

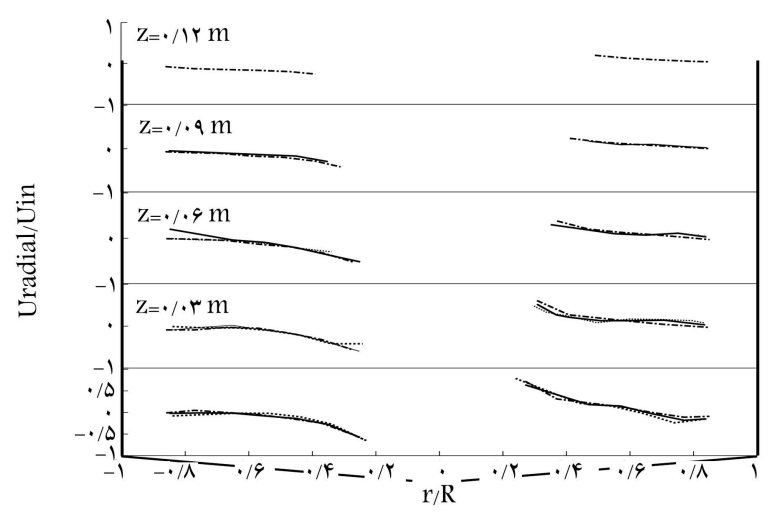

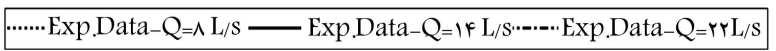

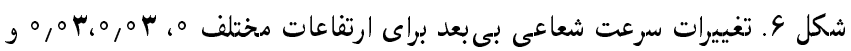

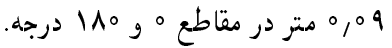

است. [0] در شكل V، خطوط جريان در دقاطع دختلف با توجه به سرعتهاى اندازهيرى شده ترسيم شده است. جهت خطوط جر يان در دقاطع بر و اله

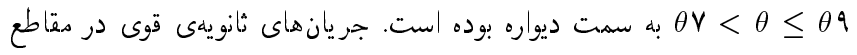
عرضى شكل مىكيرد، جايىكه جهت جريان تغيير مىكند (A)

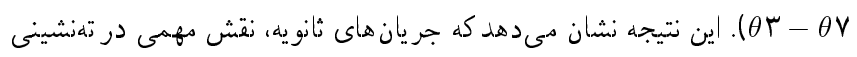
ذرات رسوبى در مقاطع ه س جهت خطوط جريان و شكلكيرى گردابه تا حدى مشابه با نتايج كشاورزى و غيثى

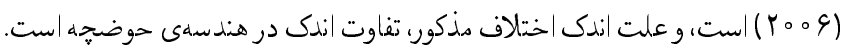

\section{F F.F} I.f.F

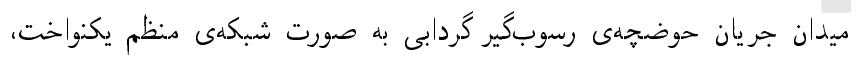

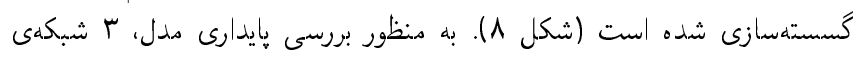

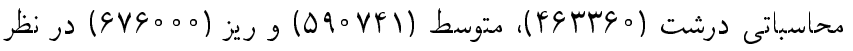

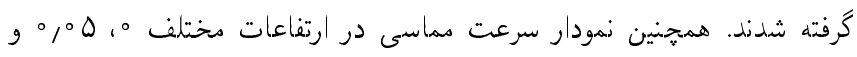

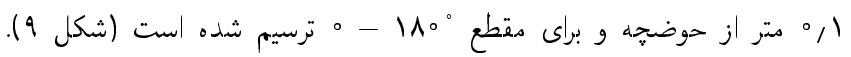

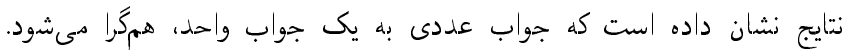

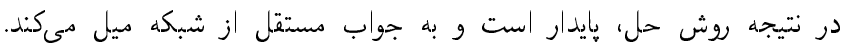

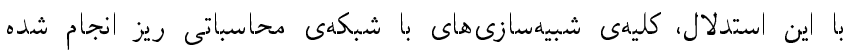

براى بررسى صحت مدلسازى عددى، سرعت مماسى به دست آمده از

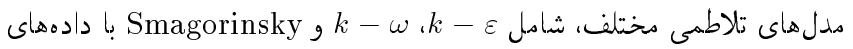

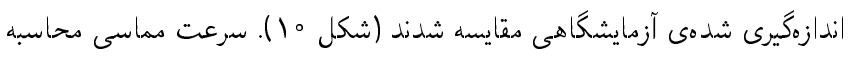

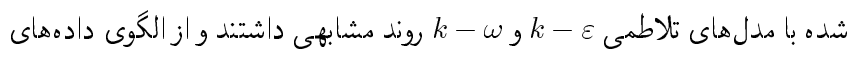

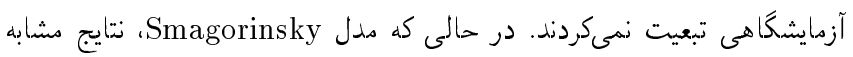

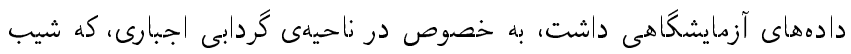

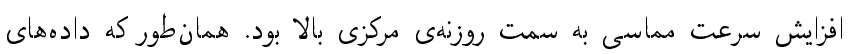

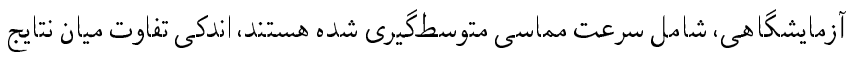

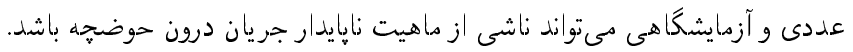




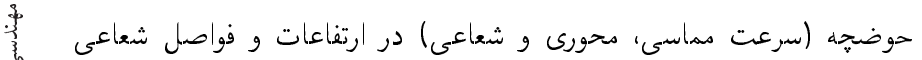

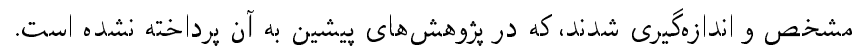

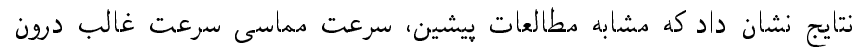

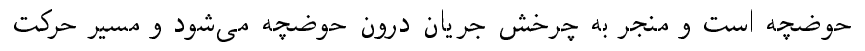

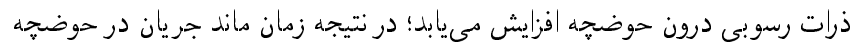

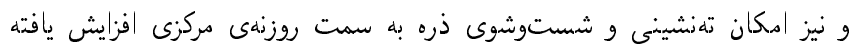

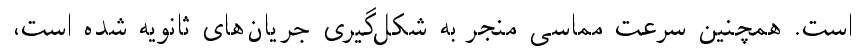

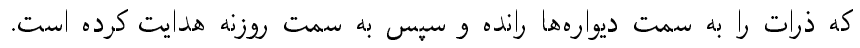

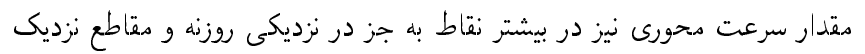

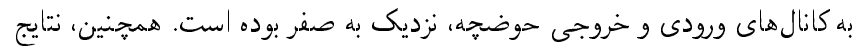

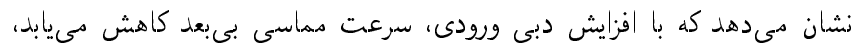

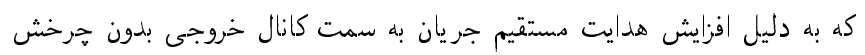

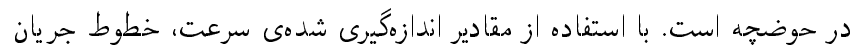

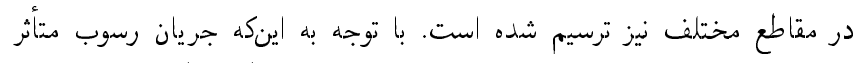

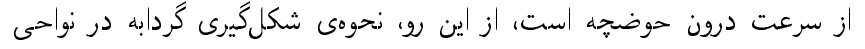

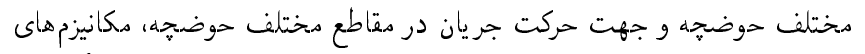

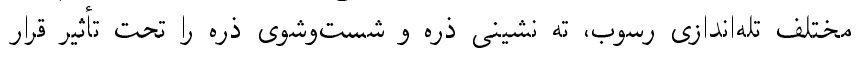
مدلسازى عددى با استفاده از مدلهاى تلاطمى RANS شامل مدلهاى

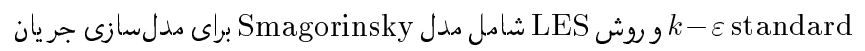

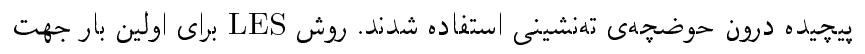

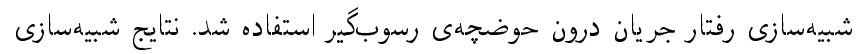

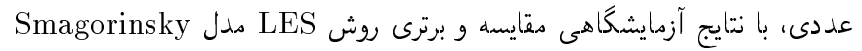

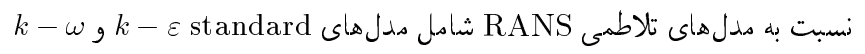

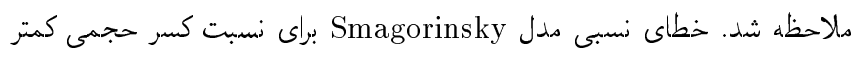

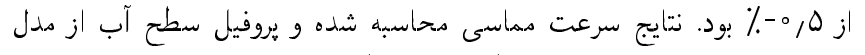

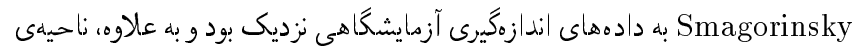

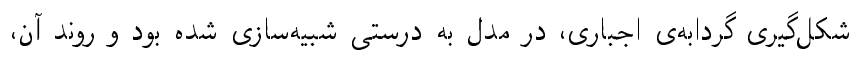

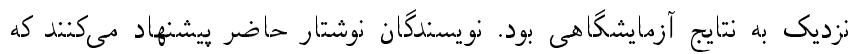

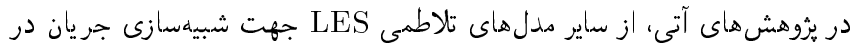
حوضجهى رسوبكير استفاده شود.

$$
\text { تشكر و قدردانى }
$$

نويسندان نوشتار حاضر كمال تشكر و قدردانى را از مركز محاسبات سريع دانشكاه

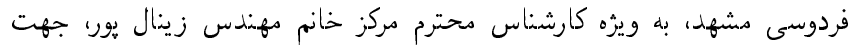

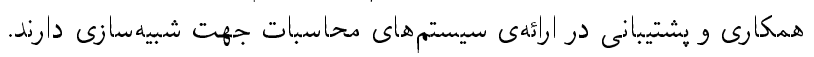

\section{فهرست علائم}

证: Qout Q

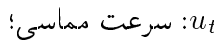

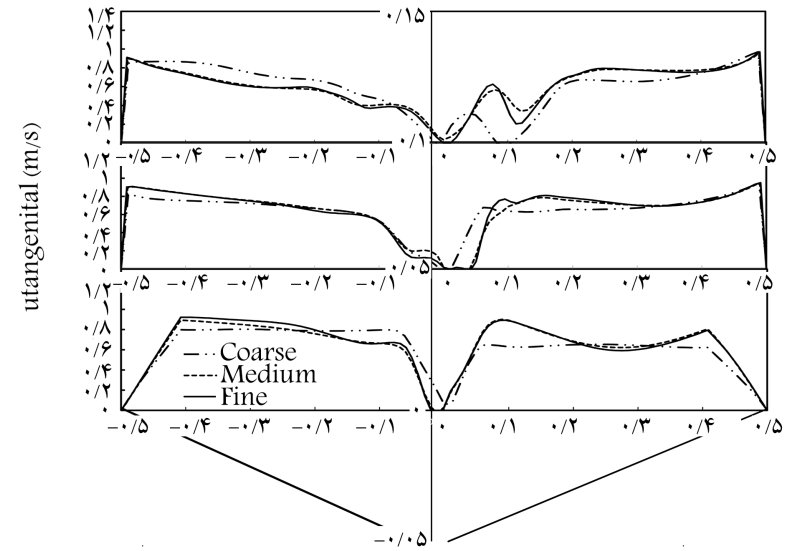

شكل 9.

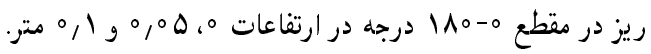

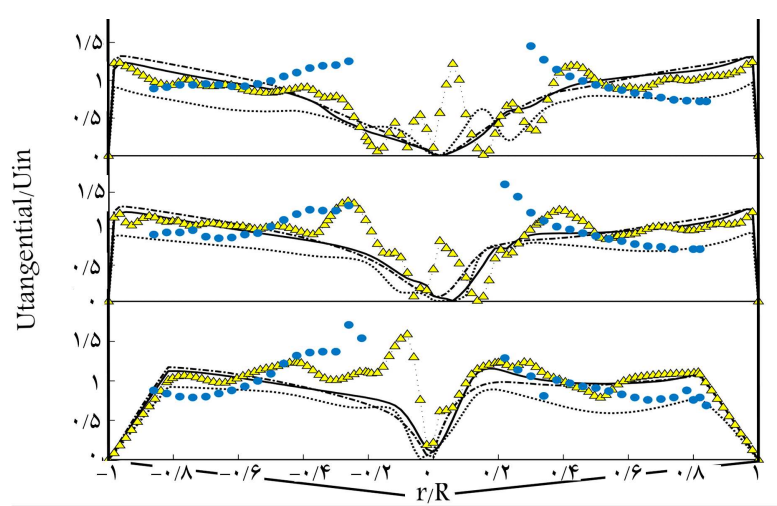

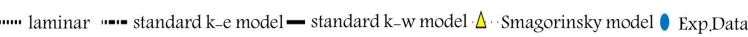

شكل 1 ـ مقايسهى سرعت مماسى محاسبه شده از مدلهاى تلاطمى با دادههاى

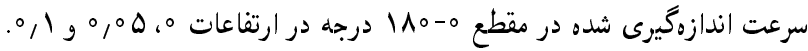
جدول r. نسبت كسر حجمى مدلهاى تلاطمى مختلف.

\begin{tabular}{|c|c|c|c|c|}
\hline$R E \%$ & $\left(Q_{o} / Q_{i n}\right) \%$ & $Q$ o & $Q_{\text {in }}$ & \\
\hline - & $\mid r, q \wedge$ & r,VO & $r l, 1$ & دادههاى آزمايشكاهى \\
\hline 1,0 & $r 0,09$ & $\varphi, r \Lambda$ & $r l, 1$ & laminar \\
\hline$I V, 0$ & $r v, v q$ & 9,09 & $r 1,1$ & $k-\varepsilon$ standard \\
\hline 10,1 & $r \Delta, r \wedge$ & 0,94 & $r 1,1$ & $k-\omega$ \\
\hline$-0,0$ & $|r, 1|$ & $r, q 4$ & $r !, \Lambda$ & Smagorinsky \\
\hline
\end{tabular}

(.F.F

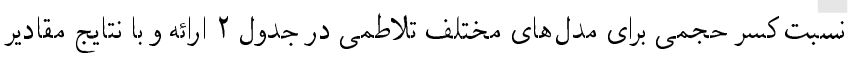

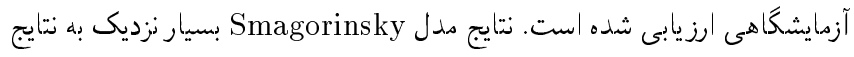

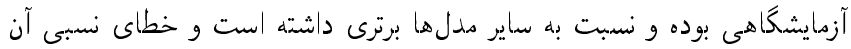
براى تمام دبى ها كمتر از هره - بـ بوده است.

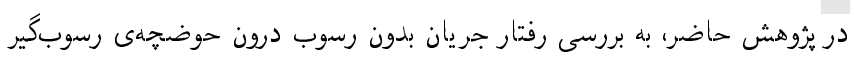

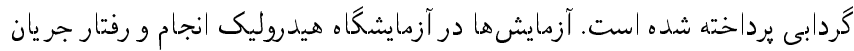

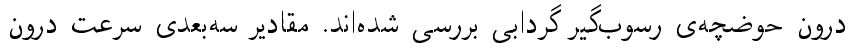




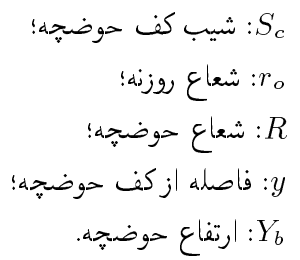

يانوشتها

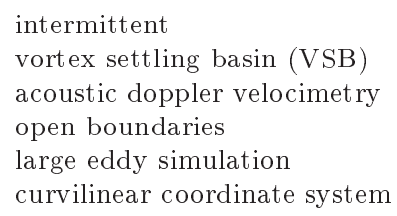

\section{منابع (References)}

1. Ziaei, AN. "Generalized three-dimensional curvilinear numerical modeling of laminar and turbulent free-surface flows in a vortex settling basin", PhD Thesis, Shiraz University, Shiraz, Iran (2007).

2. Anwar, H.O. "Turbulent flow in a vortex", J. Hydraul. Res., 7, pp. 1-29(1969).

3. Sullivan, R.H. "The swirl concentrator as a combined sewer over-flow regulatory facility", Report No: EPA-R2-72-008, U.S. Environmental Protection Agency, Washington, D.C. (1972).

4. Smisson, B.S. "Design, construction and performance of vortex overflows. Proc Symposium on Storm Sewage Overflows", Institution of Civil Engineers, London, England, pp. 99 (1967).

5. Salakho, F.S. "Rational designs and methods of hydraulic calculations of load-controlling water intake structures for mountain rivers", in Proc. 9th Congr. ICID, Moscow, Soviet Union, pp. 151-161 (1975).

6. Curi, K.V. E sen II and Velioglu SG. "Vortex type solid liquid separator", Progress Water Tchnol, 7, pp. 183-190 (1979)

7. Paul, T.C. "Circulation chamber sediment extractor", Report OD 58, Hydraulics (1983).

8. cecen, K. and Bayazit, M. "Some laboratory studies of sediment controlling structures", Proc. 9th Cong. ICID, Moscow, Soviet Union, pp. 107-111 (1975).

9. Chrysostomou, V. "Vortex-type settling basin", Thesis Presented To The University of Southampton, At Southampton, England, In Partial Fulfillment Of The Requirements For The Degree Of Master Of Science (1983).

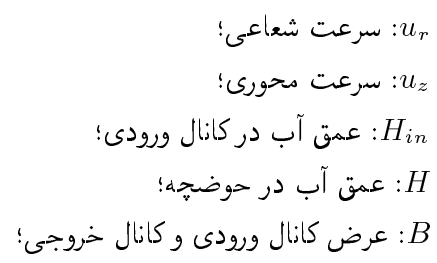

10. Ogihara, H. and Sakaguchi, S. "New system to separate the sediments from the water flow by using the rotating flow", In Proc., 4th Cong. Of the Asian and Pacific Division, IAHR, Chiang Mai, Thailand, pp. 753-766 (1984).

11. Paul, T.C. "Designing circulation chamber sediment extracter", in Report No. OD 91, Hydraulic Research Limited, Wallingford, UK (1988).

12. Paul, T.C. and Dhillon, G.S.X. "Analytical models for free-surface flow air-core vortex", In press with Indian Journal of Power and River Valley Development (1983).

13. Paul, T.C., Sayal, S.K., Sakhanja, V.S. and et al. "Vortex set tling chamber design considerations", In Journal of Hydraulic Engineering, 117(2), pp. 172-189 (1991).

14. Ziaei, A.N. "Study on the efficiency of vortex settling basin (VSB) by modeling", In MSc dissertation, Shiraz University, Shiraz, Iran (2000).

15. Athar, M., Kothyari, U.C. and Garde, R.J. "Sediment removal efficiency of vortex chamber type sediment extractor", J. Hydraul. Eng, 128, pp. 1051-1059 (2002).

16. Nguyen, Q.T. "Effect of deflectors on removal efficiency of a deep-depth vortex chamber sediment extractor", 12 th conference on science \& technology, Hochi Minh, Vietnamese (2011).

17. Saneie, M., Asefi, M. and Smaeeli, K. "Experimental studies on increasing sedimentation efficiency of vortex settling basin by using submerged vanes", Journal of Watershed Engineering and Management, 5(3), pp.224232 (2013).

18. Pishgar, R. and Keshavarzi, A.R. "Investigation of the effect of two series connected vortex settling basins (VSBs) on the efficiency of sediment xtraction", Journal of Iran-Watershed Management Science \& Engineering, 11(37), pp.65-69 (2017).

19. Julien, P.Y. "Motion of a sediment particle in Rankinecombined vortex", Report CED-84PYJ6, Colorado State University, Fort Collins (1985).

20. 20. Vatistas, GH. "Analysis of fine particle concentration in a combined vortex?, J. Hydraul. Res. Proc., 27, pp. 417-426 (1989).

21. Rea, Q. "Secondary currents within the circulation chamber sediment extractor", MS Thesis, University of Southampton, Southampton, UK (1984).

22. Zhou, Z., Wang, C. and Hou, J. "Model study on flushing cone with strong spiral flow", Proc. 4th Int. Symposium on River Sedimentation, Beijing, pp. 1213-1219 (1989). 
23. Chapokpour, J., Farhoudi, J. and Amiri Tokaldani, E. "Majedi-Asl M. flow visualization in vortex chamber", Journal of Civil Engineering and Urbanism, 2(1), pp. 26-34 (2011).

24. Lewis, N.S. "The use of an inverted small diameter hydrocyclone to classify particles of sand and gravel", Group Ordinary, Department of Geology, University of Bristol, Bristol, England (1981).

25. Luyckx, G. and Berlamont, J. "Removal efficiency of swirl/vortex separators", Urban Water Journal, 1(3), pp. 251-260 (2004).

26. Keshavarzi, A.R. and Gheisi, A.R. "Trap efficiency of vortex settling basin for exclusion of fine suspended particles in irrigation canals", J. Irrig. Drain. Engng., 55(4), pp. 419-434 (2006).

27. Athar, M., Kothyari, U.C. and Garde, R.J. "Distribution of sediment concentration in the vortex chamber type sediment extractor", J. Hydraul. Res., 41, pp. 427-438 (2003).

28. Ziaei, A.N., Mc Donough, J.M., Emdadand, H. and et al. "Using vorticity to define conditions at multiple open boundaries for simulating ow in a simplified vortex settling basin", Int. J. Numer. Meth. Fluids, 54, pp. 1-28 (2007).

29. Chan, D.J. and Quang, T.N. "Discharge coefficient for a warwe flow through a bottom orifice conical Hopper", J. of Irrig. and Drain. Eng., 8(136), pp. 572-567 (Aug., 2010).

30. Ansar, M.A. and Athar, M. "Artificial neural networks approach for estimation of sediment removal efficiency of vortex", Journal of Hydraulic Engineering, 19(1), pp. 38-48 (2013)

31. Haji Ahmadi, A., Saneie, M. and Azhdari moghdam, M. "Laboratory investigation of the effect of the diameter size of orifice on the performance of curvature submerged vanes with 450 radial section in efficiency of vortex settling basins", Watershed Management Research (Pajouhesh \& Sazandegi), 103, pp. 74-82 (2014) (In persian).

32. Chapokpour, J., Ghasemzadeh, F. and Farhoudi, J. "The Numerical Investigation on Vortex Flow Behavior Using FLOW-3D", J. WASJ, 3(1), pp. 105-113 (2012).

33. http://www.flow3d.com/resources/flow3d-technical papers-1.html.

34. Slack, MD. And Boysan, F. "Advances in cyclone modeling using unstructured grids", Scandinavian FLUENT UGM Gothenburg (1988).

35. Statie, E., Salcudean, M., Gartshore, I. and et al. "The influence of hydrocyclone geometry on separation and fibre classification", Filtration and Separation, 38(6), pp. 36-41 (2001).

36. Derksen, J.J., Sundaresan, S. and Van den Akker, H.E.A. "Simulation of mass-loading effects in gas-solid cyclone separators", J. of Powder Technology, 163, pp. 59 (2006).

37. Derksen, J.J., Sundaresan, S. and Van den Akker, H.E.A. "Two-way coupled large-eddy simulations of the gassolid flow in cyclone separators", AIChE Journal, 54, pp. 872 (2008).

38. Talbi, K., Nemouchi, Z., Donnot, A. and et al. "An experimental study and a numerical simulation of the turbulent flow under the vortex finder of a cyclone separator", Journal of Applied Fluid Mechanics, 4(1), pp. 69-75 (2009).

39. Utikar, R., Darmawan, N., TadeL, M. and et al. "Hydrodynamic Simulation of Cyclone Separators", In Computational Fluid Dynamics, Intec open publisher, pp. 241266 (2010).

40. José de Souza, F, de Vasconcelos Salvo, and Alves de Moro Martins, D. "Large eddy simulation of the gasparticle flow in cyclone separators", J. of Separation and Purification Technology, 94, pp. 61-70 (2012).

41. Saidi, M., Maddahian, R., Farhanieh, B. and et al. "Modeling of flow field and separation efficiency of a deoiling hydrocyclone using large eddy simulation", International Journal of Mineral Processing, 112-113, pp. 84-93 (2012).

42. Mikulcic, H., Vujanovi, M., Sami Ashhab, M. and et al. "Large eddy simulation of a two-phase reacting swirl flow inside a cement cyclone", J. of Energy, 75, pp. 89-96 (2014).

43. Wilcox, D.C. "Turbulence Modeling for CFD", $D C W$ Industries Inc., La Canada, CA (1993).

44. Rodi, W., Constantinescu, G. and Stoesser, T. "LargeEddy Simulation in Hydraulics?, CRC Press/Balkema, P.O. Box 11320,2301 EH Leiden, The Netherlands (2013).

45. He, P., Salcudean, M., Gartshore, I.S. and et al. "Mulitgrid calculation of fliud flows in complex 3D geometries using curviliear grids", Journal of Computers And Fli$u d s$, 25(4), pp. 395-419 (1996).

46. Raoufi, A., Shams, M., Farzaneh, M. and et al. "Numerical simulation and optimization of fluid flow in cyclone vortex finder", Journal of Chemical Engineering and Processing, 47(1), pp. 128-137 (2008). 